\title{
Isometric immersions of Riemannian products revisited
}

\author{
J. L. BarbosA, M. DajCzer AND R. TOJeiro
}

Let $M^{n}=M_{1}^{n_{1}} \times M_{2}^{n_{2}}$ be a Riemannian product of two connected complete Riemannian manifolds. Assume $\operatorname{dim} M_{i}^{n_{i}}=n_{i} \geq 2,1 \leq i \leq 2$, and that no $M_{i}$ either is flat everywhere or contains an "Euclidean strip", that is, an open submanifold which is isometric to the Riemannian product $I \times \mathbf{R}^{n_{i}-1}$, where from now on $I \subset \mathbf{R}$ denotes an open interval. Under these assumptions and based on earlier work due to Moore ([Mo]), it was proved in a beautiful paper by Alexander and Maltz ([A-M]) that any isometric immersion $f: M^{n} \rightarrow \mathbf{R}^{n+2}$ is a Riemannian product of hypersurface immersions. This means that there exist an orthogonal factorization $\mathbf{R}^{n+2}=\mathbf{R}^{n_{1}+1} \times \mathbf{R}^{n_{2}+1}$ and isometric immersions $f_{i}: M_{i}^{n_{i}} \rightarrow \mathbf{R}^{n_{i}+1}, 1 \leq i \leq 2$, such that $f\left(x_{1}, x_{2}\right)=\left(f_{1}\left(x_{1}\right), f_{2}\left(x_{2}\right)\right)$. This outstanding global theorem proved for any number of factors whenever the codimension equals that number, has been for a long time (cf. $\left[\mathrm{D}-\mathrm{G}_{2}\right]$ ) the only known global rigidity result for codimension higher than one (cf. $\left[\mathrm{Sa}_{2}\right],\left[\mathrm{D}-\mathrm{G}_{1}\right]$ ).

The main goal of this paper is to provide an understanding of the possible cases for which $f: M^{n}=M_{1}^{n_{1}} \times M_{2}^{n_{2}} \rightarrow \mathbf{R}^{n+2}$ may fail to be a Riemannian product of hypersurface immersions. An explicit example of this situation was given in ([A-M]). In fact, with no further assumptions on the $M_{i}$ 's than completeness, we are able to prove the following result:

THEOREM 1. Let $f: M^{n}=M_{1}^{n_{1}} \times M_{2}^{n_{2}} \rightarrow R^{n+2}, n_{i} \geq 2$, be an isometric immersion of a complete connected Riemannian manifold where no factor is everywhere flat. Then there is a dense open subset each of whose points lies in a product neighborhood $U=U_{1} \times U_{2}$, with $U_{j} \subset M_{j}^{n_{j}}$ open, such that $f_{U}: U_{1} \times U_{2} \rightarrow \mathbf{R}^{n+2}$ is one of the following types:

(i) $f_{U}$ is a Riemannian product of immersions.

(ii) Each $U_{i}$ is isometric to $I_{i} \times \mathbf{R}^{n_{i}-1}, 1 \leq i \leq 2$, and $f_{U}=g \times I d$, where $g$ : $I_{1} \times$ $I_{2} \rightarrow \mathbf{R}^{4}$ is an isometric immersion and Id: $\mathbf{R}^{n-2} \rightarrow \mathbf{R}^{n-2}$ is the identity map.

(iii) Only one $U_{j}$ is isometric to $I_{j} \times \mathbf{R}^{n_{j}-1}$ and $f_{U}=\tilde{f} \times I d:\left(U_{i} \times I_{j}\right) \times$ $\mathbf{R}^{n_{j}-1} \rightarrow \mathbf{R}^{n+2}, i \neq j$, where Id: $\mathbf{R}^{n_{j}-1} \rightarrow \mathbf{R}^{n_{j}-1}$ is the identity map and $\tilde{f}: U_{i} \times I_{j} \rightarrow \mathbf{R}^{n_{i}+3}$ is a composition $\tilde{f}=h \circ g$ of isometric immersions $g: U_{i} \times I_{j} \rightarrow V, V \subset \mathbf{R}^{n_{i}+2}$ open, and $h: V \rightarrow \mathbf{R}^{n_{i}+3}$. 
For types (i) we either have a product of hypersurfaces or one of the factors is totally geodesic. Types (ii) and (iii) are not disjoint since the immersions $g$ in (ii) may in fact be a composition. A complete local classification of flat surfaces in $\mathbf{R}^{4}$ which are nowhere compositions has been recently provided in [C-D]. The example in $[A-M]$ attaches immersions of types (i) and (iii).

We-also study the case where one of the factors of $M^{n}$ is everywhere flat. In this situation we have the result below for whose statement we first have to establish some definitions.

Given an isometric immersion $f: M^{n} \rightarrow \mathbf{R}^{N}$, we denote by $N_{1}^{f}(x)$ the first normal space of $f$ at $x \in M^{n}$ given by

$$
N_{1}^{f}(x)=\operatorname{span}\left\{\alpha_{f}(X, Y): \forall X, Y \in T_{x} M\right\}
$$

where $\alpha_{f}: T_{x} M \times T_{x} M \rightarrow T_{x} M^{\perp}$ stands for the vector valued second fundamental form. We say that $f$ is 1-regular if the subspaces $N_{1}(x)$ form a subbundle of the normal bundle.

An isometric immersion $f: N^{n+m} \rightarrow \mathbf{R}^{N}$ is called an $m$-cylinder whenever there exists a Riemannian manifold $M^{n}$ such that $N^{n+m}, \mathbf{R}^{N}$ and $f$ have orthogonal factorizations $N^{n+m}=M^{n} \times \mathbf{R}^{m}, \mathbf{R}^{N}=\mathbf{R}^{N-m} \times \mathbf{R}^{m}$ and $f=\tilde{f} \times I d$, where $\tilde{f}: M^{n} \rightarrow$ $\mathbf{R}^{N-m}$ is an isometric immersion and $I d: \mathbf{R}^{m} \rightarrow \mathbf{R}^{m}$ is the identity map.

THEOREM 2. Let $M^{n}$ be a complete connected Riemannian manifold of nonnegative Ricci curvature without flat points and let $f: M^{n} \times \mathbf{R}^{m} \rightarrow \mathbf{R}^{n+m+2}$ be a 1-regular isometric immersion. Then $f$ is either an $m$-cylinder or it is an $(m-1)$-cylinder,

$$
f=\tilde{f} \times I d:\left(M^{n} \times \mathbf{R}\right) \times \mathbf{R}^{m-1} \rightarrow \mathbf{R}^{n+m+2}
$$

and there exist a flat Riemannian manifold $N_{0}^{n+2}$ and isometric $g: M^{n} \times \mathbf{R} \rightarrow N_{0}^{n+2}$ and $h: N_{0}^{n+2} \rightarrow \mathbf{R}^{n+3}$ such that $\tilde{f}=h \circ g$ is a composition.

Furthermore, when $M^{n}$ is simply connected we can take $N_{0}^{n+2}$ in the latter case to be an open subset of $\mathbf{R}^{n+2}$ and, then $\mathbf{g}=\tilde{\mathbf{g}} \times I d$, where Id: $\mathbf{R} \rightarrow \mathbf{R}$ is the identity map and $\tilde{g}: M^{n} \rightarrow \mathbf{R}^{n+1}$ is an embedding whose image is a convex hypersurface.

The above result is false without the assumption of 1-regularity. Counterexamples can easily be constructed following the procedure given by Henke ([He]). For $m=1$, a weaker result but without our 1-regularity assumption has been given by Noronha ([No]).

The paper is organized as follows. In $\$ 1$ we review from [Mo] and [A-M] basic facts on isometric immersions of Riemannian products. In $\$ 2$ we prove a local result on isometric immersions $f: M^{n-1} \times I \rightarrow \mathbf{R}^{n+2}$ which is a crucial step in the proof of 
Theorem 1 and also of independent interest. Finally, in $\$ 3$ we present the proofs of our main results where we make strong use of the fine arguments in [A-M].

\section{§1. Preliminaries}

Let $f: M^{n} \rightarrow \mathbf{R}^{n+k}$ be an isometric immersion. Recall that the relative nullity space of $f$ at $x \in M^{n}$ is defined by

$$
\Delta_{x}=\left\{X \in T_{x} M: \alpha_{f}(X, Y)=0, \forall Y \in T_{x} M\right\}
$$

Then, by the Gauss equation, $\Delta_{x}$ is contained in the nullity space of $f$ at $x \in M^{n}$ given by

$$
N_{x}=\left\{X \in T_{x} M: R(X, Y)=0, \forall Y \in T_{x} M\right\},
$$

where $R$ denotes the curvature tensor of $M^{n}$.

A classical inequality due to Chern and Kuiper says that the index of nullity $\mu(x)=\operatorname{dim} N_{x}$ and the index of relative nullity $v(x)=\operatorname{dim} \Delta_{x}$ verify

$$
0 \leq \mu(x)-v(x) \leq k
$$

Let $M^{n}=M_{1}^{n_{1}} \times M_{2}^{n_{2}}, n_{i} \geq 2$, be a Riemannian product. The following sharpened version of Chern-Kuiper's inequality derived in [A-M] holds.

LEMMA 3. Let $f: M^{n}=M_{1}^{n_{1}} \times M_{2}^{n_{2}} \rightarrow \mathbf{R}^{n+2}$ be an isometric immersion. At any point $x=\left(x_{1}, x_{2}\right) \in M^{n}$, we have

$$
0 \leq \mu(x)-v(x) \leq k^{\prime}(x)
$$

where $k^{\prime}(x)$ is the number of factors $M_{i}^{n_{i}}$ flat at $x_{i}$.

Denote by $\pi_{i}$ the orthogonal projection of $T_{x} M$ onto $T_{x_{i}} M_{i}$ and by $M_{i}^{n_{i}}(x)$ the copy of $M_{i}^{n_{i}}$ through $x=\left(x_{1}, x_{2}\right) \in M^{n}$. The relative nullity and the nullity spaces of $\left.f\right|_{M_{i}^{n_{i}(x)}}$ at $x$ will be denoted by $\Delta_{i x}$ and $N_{i x}$ respectively. Then, it is not difficult to show that $N_{i x}=N_{x} \cap \pi_{i}\left(T_{x} M\right), \Delta_{i x}=\Delta_{x} \cap \pi_{i}\left(T_{x} M\right)$ and

$$
\Delta_{1 x} \oplus \Delta_{2 x} \subset \Delta_{x} \subset \pi_{1}\left(\Delta_{x}\right) \oplus \pi_{2}\left(\Delta_{x}\right) \subset N_{x}=\pi_{1}\left(N_{x}\right) \oplus \pi_{2}\left(N_{x}\right)=N_{1 x} \oplus N_{2 x}
$$

with equality holding at the first inclusion if and only if it holds at the second. A simple example is provided in [A-M] where equality does not hold. If it does we say 
as in [A-M] that $\Delta_{x}$ conforms to the product structure of $M^{n}$. A first and fundamental step in the proof of the main theorem in [A-M] was to show that this is always the case if the $M_{i}^{n_{i}}$ 's are complete, unless one of them is everywhere flat.

LEMMA 4. Let $f: M^{n}=M_{1}^{n_{1}} \times M_{2}^{n_{2}} \rightarrow \mathbf{R}^{n+2}$ be an isometric immersion of a complete connected Riemannian manifold. Then the relative nullity spaces of $f$ conform to the product structure of $M^{n}$ unless one of the factors is everywhere flat.

Next, we state a more basic lemma due to Moore ([Mo]). Following [A-M] we will say that condition $\alpha_{f}\left(X_{1}, X_{2}\right)=0$ holds at $x \in M^{n}$ if this equation holds for any $X_{1} \in T_{x_{1}} M_{1}, X_{2} \in T_{x_{2}} M_{2}$. Also, given an open subset $S$ of $M_{i}(x)$, a point $y$ is said to be visible along $S$ from $x$ if there is a geodesic $\gamma$ satisfying $\gamma(0)=x$, $\gamma(b)=y, \gamma(s) \in S$ and $\gamma^{\prime}(s) \in \Delta_{i \gamma(s)}$ for $0 \leq s \leq b$.

LEMMA 5. For an isometric immersion $f: M^{n}=M_{1}^{n_{1}} \times M_{2}^{n_{2}} \rightarrow \mathbf{R}^{n+2}$ the following assertions are true:

(i) If $\alpha_{f}\left(X_{1}, X_{2}\right)=0$ holds everywhere on $M^{n}$, then $f$ is a product of immersions.

(ii) If $M_{i}^{n_{i}}$ is not flat at $x_{i}, 1 \leq i \leq 2$, then $\alpha_{f}\left(X_{1}, X_{2}\right)=0$ holds at $x=\left(x_{1}, x_{2}\right)$.

(iii) Let $S$ be an open subset of $M_{1}^{n_{1}}(x)$ on which the spaces $\Delta_{1 x}$ have constant dimension. If a point where $\alpha_{f}\left(X_{1}, X_{2}\right)=0$ holds is visible along $S$ from $x$, then $\alpha_{f}\left(X_{1}, X_{2}\right)=0$ holds at $x$ also.

We conclude this section with a well known characterization of complete Euclidean cylinders due to Hartman ([Ha]). Recall that in a Riemannian manifold a line is a complete goedesic such that every subarc is minimizing.

LEMMA 6. Let $f: M^{n} \rightarrow \mathbf{R}^{N}$ be an isometric immersion of a connected complete Riemannian manifold with nonnegative Ricci curvature such that $f\left(M^{n}\right)$ contains $m$ linearly independent lines through one point. Then $f$ is an $m$-cylinder.

\section{§2. The local result}

THEOREM 7. Let $M^{n-1}$ be a connected Riemannian manifold without flat points and let $f: N^{n}=M^{n-1} \times I \rightarrow \mathbf{R}^{n+2}$ be an isometric immersion such that $\alpha_{f}\left(X_{1}, X_{2}\right)=0$ fails everywhere. Then there exist a flat Riemannian manifold $N_{0}^{n+1}$ and isometric immersions $g: N^{n} \rightarrow N_{0}^{n+1}$ and $h: N_{0}^{n+1} \rightarrow \mathbf{R}^{n+2}$ such that $f=h \circ g$ is a composition. Furthermore, $N_{0}^{n+1}$ may be taken to be an open subset of $\mathbf{R}^{n+1}$ when $f$ is an embedding. 
In what follows $Z$ will always denote a unit vector field tangent to $I$.

LEMMA 8. Assume that $M^{n-1}$ is nowhere flat and that $f: N^{n}=M^{n-1} \times$ $I \rightarrow \mathbf{R}^{n+2}$ verifies $\alpha_{f}(Z, Z) \neq 0$ everywhere. Then there exists a smooth unitary normal vector field $\xi$ such that everywhere $\operatorname{rank} A_{\xi}=1, \operatorname{Im} A_{\xi} \notin T M$ and $A_{\eta} Z=0$ for any section $\eta \in L$ of the smooth normal line bundle with fibers orthogonal to $\xi$.

Proof. Define $\xi \in T N^{\perp}$ by $\alpha_{f}(Z, Z)=\mu \xi$ and let $\eta \in L$ be a smooth unitary local section. Since $\left\langle A_{\eta} Z, Z\right\rangle=0$, there exist $X_{1}, \ldots, X_{n-1} \in T M$ such that $X_{n-1}, \ldots, X_{1}, Z$ is an orthonormal frame of $T N$ with respect to which $A_{\eta} Z=\gamma X_{1}, A_{\xi} Z=\mu Z+\lambda_{1} X_{1}+\lambda_{2} X_{2}$, where $\mu \neq 0$ by assumption. We have:

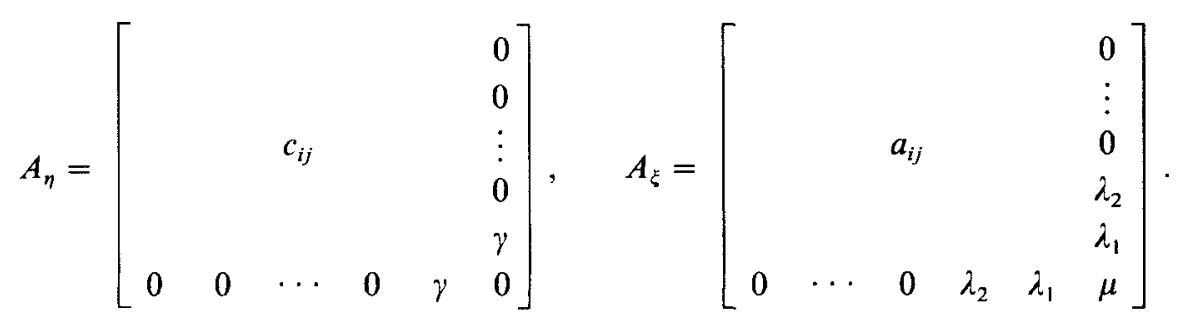

From $\left\langle R\left(X_{i}, Z\right) Z, X_{j}\right\rangle=0$ and the Gauss equations, we get

$$
\mu a_{i j}=\left\langle A_{\eta} Z, X_{i}\right\rangle\left\langle A_{\eta} Z, X_{j}\right\rangle+\left\langle A_{\xi} Z, X_{i}\right\rangle\left\langle A_{\xi} Z, X_{j}\right\rangle
$$

Thus

$$
a_{i j}=0 \quad \text { for } i \geq 3, \quad \mu a_{11}=\gamma^{2}+\lambda_{1}^{2}, \quad \mu a_{12}=\lambda_{1} \lambda_{2}, \quad \mu a_{22}=\lambda_{2}^{2}
$$

The Gauss equations and $\left\langle R\left(X_{i}, Z\right) X_{1}, X_{k}\right\rangle=0$ yield

$$
\gamma c_{i k}=C_{1 i}\left\langle A_{\eta} Z, X_{k}\right\rangle+\lambda_{1} a_{i k}-a_{1 i}\left\langle A_{\xi} Z, X_{k}\right\rangle=0 .
$$

Hence,

$$
\gamma c_{i 2}+\lambda_{1} a_{i 2}-\lambda_{2} a_{1 i}=0, \quad \gamma c_{i k}+\lambda_{1} a_{i k}=0 \quad \text { for } k \geq 3
$$

Using (1), we get

$$
\left\{\begin{array}{l}
\gamma c_{12}=\lambda_{2} a_{11}-\lambda_{1} a_{12}=\lambda_{2} \gamma^{2} / \mu \\
\gamma c_{22}=\lambda_{2} a_{12}-\lambda_{1} a_{22}=0 \\
\gamma c_{i k}=0 \text { for } k \geq 3
\end{array}\right.
$$


We claim that $\gamma=0$. Otherwise, we conclude from (2) that $c_{12}=\lambda_{2} \gamma / \mu, c_{22}=0$ and $c_{i k}=0$ for $k \geq 3$. Therefore,

$$
A_{\eta}=\left[\begin{array}{ccccc}
0 & \cdots & & & 0 \\
\vdots & & & & \vdots \\
& & 0 & \lambda_{2} \gamma / \mu & 0 \\
& & \lambda_{2} \gamma / \mu & c_{11} & \gamma \\
0 & \cdots & 0 & \gamma & 0
\end{array}\right], A_{\xi}=\left[\begin{array}{ccccc}
0 & \cdots & & & 0 \\
\vdots & & & & \vdots \\
& & \lambda_{2}^{2} / \mu & \lambda_{1} \lambda_{2} / \mu & \lambda_{2} \\
& & \lambda_{1} \lambda_{2} / \mu & \left(\gamma^{2}+\lambda_{1}^{2}\right) / \mu & \lambda_{1} \\
0 & \cdots & \lambda_{2} & \lambda_{1} & \mu
\end{array}\right] .
$$

Thus we have for the sectional curvatures

$$
K\left(X_{1}, X_{2}\right)=-\frac{\lambda_{2}^{2} \gamma^{2}}{\mu^{2}}+\frac{\lambda_{2}^{2}}{\mu}\left(\frac{\gamma^{2}+\lambda_{1}^{2}}{\mu}\right)-\frac{\lambda_{1}^{2} \lambda_{2}^{2}}{\mu^{2}}=0, \quad K\left(X_{i}, X_{j}\right)=0, \quad 2 \leq i, j \leq n,
$$

which contradicts our assumption that $M^{n-1}$ is nowhere flat and proves our claim. Notice that $A_{\xi}$ is given by (3) regardless of $\gamma$ being zero or not. Hence rank $A_{\xi}=1$ and this concludes the proof.

Proof of Theorem 7: In what follows we represent by $\nabla$ the Riemannian connection in $N^{n}$ and by $\nabla^{\perp}$ the induced connection in the normal bundle. By assumption, at any $(x, t) \in N^{n}$ there exists $X \in T_{x} M$ for which $\alpha_{f}(X, Z) \neq 0$. On the other hand, the sectional curvature $K(X, Z)$ vanishes. We conclude from the Gauss equation that $\alpha_{f}(Z, Z) \neq 0$ everywhere. In particular, Lemma 8 applies.

Define $\psi: T N \rightarrow \mathbf{R}$ by $\psi(W)=\left\langle\nabla_{W}^{\frac{1}{W}} \eta, \xi\right\rangle$ where $\eta \in L$ is taken unitary. We claim that

$$
\text { ker } A_{\xi} \subset \operatorname{ker} \psi
$$

Let $X$ be any vector field tangent to $M^{n-1}$. Since $A_{\eta} Z=\nabla_{X} Z=\nabla_{Z} X=0$, the Codazzi equation for $A_{\eta}, X$ and $Z$ reduces to

$$
\nabla_{Z} A_{\eta} X=A_{\nabla_{Z} \eta} X-A_{\nabla_{X}^{\frac{1}{X} \eta}} Z=\psi(Z) A_{\xi} X-\psi(X) A_{\xi} Z
$$

Denote by $Y$ a unit vector field spanning the eigenbundle of $A_{\xi}$ corresponding to the nonzero principal curvature $\lambda$. Notice that the right hand side of the above equation is a multiple of $Y$. On the other hand, $\alpha_{f}(Z, Z) \neq 0$ implies that $\langle Z, Y\rangle \neq 0$. From this and

$$
\left\langle\nabla_{Z} A_{\eta} X, Z\right\rangle=Z\left\langle A_{\eta} X, Z\right\rangle-\left\langle A_{\eta} X, \nabla_{Z} Z\right\rangle=0
$$


we conclude that

$$
\nabla_{Z} A_{\eta} X=0
$$

Hence, taking the inner product of (5) with $Y$, we get

$$
\lambda \psi(\langle X, Y\rangle Z-\langle Z, Y\rangle X)=0
$$

Since $\langle Z, Y\rangle \neq 0$, the linear map $S:\{Z\}^{\perp} \rightarrow\{Y\}^{\perp}$ given by $S(X)=\langle X, Y\rangle Z-$ $\langle Z, Y\rangle X$ has trivial kernel. Therefore it maps $\{Z\}^{\perp}$ onto $\{Y\}^{\perp}$ and the claim follows from (7).

To conclude the proof we make use of arguments from [D-T]. Let $\pi: T \rightarrow N^{n}$ denote the line bundle whose fibers are contained in the plane bundle $\operatorname{span}\{Y\} \oplus L$ and are everywhere orthogonal to $\tilde{\nabla}_{Y} \xi=-\lambda Y+\psi(Y) \eta$. Here $\tilde{\nabla}$ stands for the connection in the ambient space. Hence, the fibers of $T$ given by span $\{\psi(Y) Y+\lambda n\}$ are nowhere tangent to $N^{n}$. Now define a hypersurface $F: T \rightarrow \mathbf{R}^{n+2}$ by

$$
F(\delta)=f(x)+\delta, \quad x=\pi(\delta)
$$

Then $F$ is an immersion when restricted to a tubular neighborhood $N_{0}^{n+1}$ of the zero section $N^{n}$ of $T$. Moreover, if $f$ is an embedding, then $N_{0}^{n+1}$ can be taken to be an open subset of $\mathbf{R}^{n+1}$ embedded in $\mathbf{R}^{n+2}$. For local sections $X \in T N$ and $\mu \in T$, we have by condition (4)

$$
\left(\tilde{\nabla}_{X} \mu, \xi\right\rangle=-\left\langle\mu, \tilde{\nabla}_{X} \xi\right\rangle=\left\langle\mu, A_{\xi} X-\psi(X) \eta\right\rangle=\langle X, Y\rangle\langle\mu, \lambda Y-\psi(Y) \eta\rangle=0 .
$$

Therefore, the Gauss map of $\left.F\right|_{N_{0}}$ is $\xi$. Since $\xi$ only depends on one parameter, the metric induced by $F$ on $N_{0}^{n+1}$ is flat.

\section{§3. The proofs of the main results}

Proof of Theorem 1: Let $X$ denote the open subset of $M^{n}$ on which condition $\alpha_{f}\left(X_{1}, X_{2}\right)=0$ fails. Then $f$ is a product of immersions by (i) of Lemma 5 on any connected open subset $U=U_{1} \times U_{2} \subset M^{n}-\bar{X}, U_{j} \subset M_{j}^{n_{j}}$. Now set

$\tilde{V}_{0}=\left\{x \in X: M_{i}^{n_{i}}\right.$ is flat at $x_{i}$ for $\left.i=1,2\right\}$ 
and, for $i=1,2$,

$$
V_{i}=\left\{x \in X: M_{i}^{n_{i}} \text { is not flat at } x_{i} \text { and } M_{j}^{n_{j}} \text { is flat at } x_{j} \text { for } j \neq 1\right\} \text {. }
$$

Then $X=\tilde{V}_{0} \cup V_{1} \cup V_{2}$ by part (ii) of Lemma 5 .

We claim that $v_{i} \equiv n_{i}-1$ on $\tilde{V}_{0} \cup V_{j}, i \neq j$.

By Lemmas 3 and 4, the sum of the codimensions of the $\Delta_{i}$ 's in the $N_{i}$ 's at $x \in X$ is $k^{\prime}(x)=1,2$. Since $v_{h}(x) \subseteq n_{h}-1,1 \leq h \leq 2$, because $\alpha_{f}\left(X_{1}, X_{2}\right)=0$ fails at $x$, our claim follows.

We show that the $V_{i}$ 's are open subsets of $M^{n}$. Given $x \in V_{i}$, let $W$ be a neighborhood of $x$ in $X$ where $v_{i}(y) \leq v_{i}(x)$ for all $y=\left(y_{1}, y_{2}\right) \in W$. Hence $W \subset V_{i}$ since $M_{i}^{n_{i}}$ is not flat at $y_{i}$.

Now let $x_{0} \in V_{i}$ and set $S=M_{j}^{n_{j}}\left(x_{0}\right) \cap V_{i}, j \neq i$. Given $x \in S$ consider a geodesic $\gamma$ tangent to $\Delta_{j}$ with $\gamma(0)=x$. Assume that $\gamma([0, b)) \subset V_{i}$ for some $b \in \mathbf{R}$. By part (iii) of Lemma 5, we have $\gamma(b) \in X$. On the other hand, since the $M_{i}$-component of $\gamma$ is constant, we conclude that $\gamma$ remains in $V_{i}$. Therefore, the leaves of $\Delta_{j}$ in $V_{i}$ are complete. A similar argument shows that the leaves of $\Delta_{1}$ and $\Delta_{2}$ in $V_{0}=$ int $\tilde{V}_{0}$ must also be complete. From the argument in ([A-M], p. 53) it follows that $S$ is isometric to $I\left(x_{0}\right) \times \mathbf{R}^{n_{j}-1}$. The same conclusion can easily be reached from Lemma 1.1 in [Ha] whose proof only uses completeness of the leaves of the relative nullity foliation.

We claim that the spaces $\mathbf{R}^{n_{j}-1}$ are parallel along any component of $V_{i}$ with $i=1,2$. As in the proof of Lemma 8 , let $Z$ denote a unit vector field tangent to the intervals and $\xi$ a unit normal vector field parallel to $\alpha_{f}(Z, Z)$. All we have to show is that $\nabla_{X} Z=0$ for all $X \in T M_{i}$. From the Codazzi equation for $A_{\xi}, X$ and $Z$, we have for any $W \in \Delta_{j}$ that

$$
\left\langle\nabla_{X} A_{\xi} Z-\nabla_{Z} A_{\xi} X, W\right\rangle=0
$$

Set $A_{\xi} Z=\lambda_{1} X_{1}+\mu Z$, where $X_{1}$ is unitary and orthogonal to $Z$. Since $\nabla_{Z} Z=0$, we get

$$
\left\langle\nabla_{Z} A_{\xi} X, W\right\rangle=-\left\langle A_{\xi} X, V_{Z} W\right\rangle=\left\langle A_{\xi} X, Z\right\rangle\left\langle Z, \nabla_{Z} W\right\rangle=0 .
$$

Hence,

$$
\left\langle\nabla_{X} A_{\xi} Z, W\right\rangle=-\left\langle A_{\xi} Z, \nabla_{X} W\right\rangle=\lambda_{1}\left\langle X_{1}, \nabla_{X} W\right\rangle+\mu\left\langle Z, \nabla_{X} W\right\rangle=0 .
$$

Because $\left\langle X_{1}, \nabla_{X} W\right\rangle=-\left\langle\nabla_{X} X_{1}, W\right\rangle=0$ and $\mu \neq 0$, we conclude that $\left\langle W, \nabla_{X} Z\right\rangle=0$ which proves our claim. 
From the claim and the above, every point of $V_{i}$ has a neighborhood of type $U \times I \times \mathbf{R}^{n_{j}-1}$, where $U$ is an open subset of $M_{i}^{n_{i}}$, and $f$ splits as a product $\tilde{f} \times I d$ with $\tilde{f}: U \times I \rightarrow \mathbf{R}^{n_{i}+3}$ an isometric embedding. We conclude from Theorem 7 that $\tilde{f}$ is as in (iii) of the statement and the remainder of the proof is straightforward.

Proof of Theorem 2: The hypothesis of $f$ being 1-regular implies that rank $N_{1}^{f}$ is constant and equal to either 1 or 2 . Assume first that rank $N_{1}^{f}=1$. By assumption rank $A_{\delta} \geq 2$ for any nonzero $\delta \in N_{1}^{f}$. It is now a standard result (see [Sp], Lemma 28 of chapter 12) that the immersion reduces codimension to one. It follows easily from Lemma 3 and 6 (cf. [Ma]) that $f$ is $m$-cylindrical.

Now assume that rank $N_{1}^{f}=2$. We have to consider two cases:

Case 1: There exists $x \in M^{n}$ such that $\alpha_{f}(Z, \tilde{Z})=0$ for all $Z, \tilde{Z}$ tangent to $\{x\} \times \mathbf{R}^{m}$ and all $y \in \mathbf{R}^{m}$. Then, the image of $\{x\} \times \mathbf{R}^{m}$ is totally geodesic in the Euclidean space and we conclude from Lemma 6 that $f$ is $m$-cylindrical.

Case 2.: It follows from Lemmas 3 and 6 that $f$ is cylindrical with respect to a hyperplane in $\mathbf{R}^{m}$. Therefore, it suffices to argue for $m=1$. In this case we are assuming that for all $x \in M^{n}$ there exists $t \in \mathbf{R}$ such that at $(x, t)$ we have $\alpha_{f}(Z, Z) \neq 0$ for $Z$ tangent to R. Fix $x$ and consider the open subset of the line $\{x\} \times \mathbf{R}$ where $\alpha_{f}(Z, Z) \neq 0$. By Lemma 8 there exist orthonormal vector fields $\xi$ and $\eta$ along the subset such that $A_{\eta} Z=0$ and rank $A_{\xi}=1$. We show that the subset is the entire line. If not, let $\bar{t}$ represent a boundary point. We have easily from (6) that $A_{\eta}$ is parallel along the open subset. Thus $A_{\eta}$ extends to the point $(x, \bar{t})$. We easily conclude that at this point $\operatorname{dim} N_{1}^{f}=1$, which is a contradiction and proves our claim.

We want to obtain here the same conclusion of Theorem 7 although we do not have the same hypothesis. Nevertheless, we argue that the above condition implies condition (4) and then the remainder of the proof there applies to our case. In fact, where $\alpha_{f}(X, Z)=0$ fails the proof of (4) is exactly the one of Theorem 7. At the other points, the conclusion is trivial using (i) of Lemma 5 . Therefore, there exist a flat Riemannian manifold $N_{0}^{n+2}$ and isometric immersions $g: M^{n} \times \mathbf{R} \rightarrow N_{0}^{n+2}$ and $h: N_{0}^{n+2} \rightarrow \mathbf{R}^{n+3}$ such that $f=h \circ g$.

Assume now that $M^{n}$ is simply connected. Then also $N_{0}^{n+2}$ is simply connected. Because it is flat it can be isometrically immersed in Euclidean space $\mathbf{R}^{n+2}$. The second fundamental form of $g$, considered into $\mathrm{R}^{n+2}$, is $A_{\eta}$. Since $A_{\eta} Z=0$, it follows from Lemma 6 that $g=\tilde{g} \times I d$, where $I d: \mathbf{R} \rightarrow \mathbf{R}$ is the identity map and $\tilde{g}: M^{n} \rightarrow \mathbf{R}^{n+1}$ is an isometric immersion whose image is a convex hypersurface by a well-known theorem of Sacksteder $\left(\left[\mathbf{S a}_{1}\right]\right)$. Since $N_{0}^{n+2}$ is just a tubular neighborhood of $g\left(M^{n}\right)$ then it can be chosen to be embedded. This completes our proof. 


\section{REFERENCES}

[A-M] S. AleXANder, R. MALTZ, Isometric immersions of Riemannian product in Euclidean space, J. Diff. Geometry 11 (1976), 47-57.

[C-D] M. Do CaRmo, M. DaJCzer, Local isometric immersion of $\mathbf{R}^{2}$ into $\mathbf{R}^{4}$, J. reine angew Math. 442 (1993), 205-219.

[D-G $\left.{ }_{1}\right]$ M. DAJCzer, D. Gromoll, Rigidity of complete Euclidean hypersurface. J. Diff. Geometry 31 (1990), $401-416$.

[D-G $\left.\mathrm{G}_{2}\right]$ M. DAJCZER, D. GROMOLL, Isometric deformations of compact Euclidean submanifolds in codimension 2. Preprint.

[D-T] M. DAJCzER, R. TojeIro, On compositions of isometric immersions, J. Diff. Geometry 36 (1992), 1-18.

[Ha] P. HARTMAN, On isometric immersions in Euclidean space of manifolds with nonnegative sectional curvatures II, Trans. Amer. Math. Soc. 147 (1970), 529-540.

[He] W. HENKE, Uber die isometrischer Fortsetzbarkeit isometrischer Immersionen der Standard-mSphäre $S^{m}\left(\subset \mathbf{R}^{m+1}\right)$ in $\mathbf{R}^{m+2}$, Math. Ann. 219 (1976), $261-276$.

[Ma] R. MAltz, Cylindricity of isometric immersions into Euclidean space, Proc. Amer. Math. Soc. 53 (1975), $428-432$.

[Mo] J. D. MOORE, Isometric immersions of Riemannian product, J. Diff. Geometry 5(1971), 159-168.

[No] M. Noronha, Nonnegatively curved submanifolds in codimension two, Trans. Amer. Math. Soc. 332 (1992), 351-364.

[Sa $\mathrm{Sa}_{1}$ R. SACKSTEDER, On hypersurfaces with no negative sectional curvatures, Amer. J. Math. 82 (1960), 609-630.

[Sa $\left.\mathrm{Sa}_{2}\right] \quad \mathrm{R}$. SACKSTEDER, The rigidity of hypersurfaces. J. Math. Mech. 11 (1962), 929-939.

[Sp] M. SPIVAK, A Comprehensive Introduction to Differential Geometry, Publish or Perish Inc., Berkeley 1979.

Universidade Federal do Ceará 60455-760 - Fortaleza - Brazil

e-mail:mate@taiba-ufc-br

Universidade Federal de Uberlândia

38400-020 - Uberlandia - Brazil

e-mail: DEMAT04@brufu.bitnet

and

IMPA-Estrada Dona Castorina 110 22460-320 - Rio de Janeiro - Brazil

e-mail: MARCOS@impa-br

Received June 21, 1993; March 28, 1994 